\title{
Probing the geometry of two-qubit state space by evolution
}

\author{
Andrzej M. Frydryszak ${ }^{1}$ (D) Maria Gieysztor ${ }^{1,2} \cdot$ Andrij Kuzmak $^{3}$ \\ Received: 12 October 2018 / Accepted: 23 January 2019 / Published online: 6 February 2019 \\ (c) The Author(s) 2019
}

\begin{abstract}
We provide explicit geometric description of state manifolds obtained from evolution governed by a four-parameter family of time-independent Hamiltonians. We cover most cases related to the real interacting two-qubit systems and discuss possible types of evolutions in terms of the defining parameters. The relevant description of the pure state spaces and their Riemannian geometry with the Fubini-Study metric is given. In particular, we analyze the modification of known geometry of quantum state manifold by the linear noncommuting perturbation of the Hamiltonian. Finally, we investigate the behavior of the entanglement for obtained families of states resulting from the unitary evolution.
\end{abstract}

Keywords Geometry of quantum state space · Fubini-Study metric · Quantum evolution $\cdot$ Compound systems $\cdot$ Entanglement

\section{Introduction}

The precise geometric description of the full state space of quantum system is crucial in studying its physical properties [1-18], especially for compound systems, where characterization of this space beyond the general property of convexity for multilevelsystems gets rather involved, even for bipartite systems. In general, such a quantum

$凶 \quad$ Andrzej M. Frydryszak

andrzej.frydryszak@ift.uni.wroc.pl

Maria Gieysztor

maria.gieysztor@ift.uni.wroc.pl

Andrij Kuzmak

andrijkuzmak@gmail.com

1 Institute of Theoretical Physics, University of Wroclaw, pl. M. Borna 9, 50-204 Wrocław, Poland

2 Faculty of Physics, Astronomy and Informatics, Nicolaus Copernicus University, Grudziadzka 5, 87-100 Toruń, Poland

3 Department for Theoretical Physics, Ivan Franko National University of Lviv, 12 Drahomanov St., L'viv 79005, Ukraine 
state space cannot be expected to form a smooth manifold. However, in the case of two-qubit system, on which we shall focus in the present work, the quantum state space is 15-dimensional convex set, and its pure state subset forms seven-dimensional sphere $S^{7}$, where the separable pure states are located within $S^{7}$ in the product of two Bloch spheres $S^{2} \times S^{2}[19]$.

On the other hand, there is an option to focus on distinguished subsets of states of quantum system, namely on orbits generated by unitary evolutions defined by physically relevant Hamiltonians which can be realized experimentally. Such a focus has been proven fruitful from various perspectives, such as the control theory [20,21], the quantum brachistochrone problem [22,23], the time-optimal evolution [5-8,21,24, 25], or the question of Zermelo navigation [26-28].

The geometry of the set of quantum states obtained as result of unitary evolutions depending on a set of parameters can be naturally studied with the use of the FubiniStudy metric [2,4,29-33], where the dimension of such Riemannian manifold is equal to the number of parameters included in the Hamiltonian. Obviously, the details of such orbits depend on applied Hamiltonian and selected initial states. Most of the interactions of a two-qubit systems can be described by the generalized Heisenberg-type interaction Hamiltonian containing anisotropic terms, which is conventionally put into the following physical form, suitable, for example, for studying quantum dot systems [10]

$$
H=J\left(\vec{S}_{1} \cdot \vec{S}_{2}+\vec{\kappa}_{\mathrm{DM}} \cdot\left(\vec{S}_{1} \times \vec{S}_{2}\right)+\vec{S}_{1} \Theta \vec{S}_{2}\right)
$$

where $\vec{S}_{j}$ are spins of $j=1,2$ of subsystems; $\vec{\kappa}_{\mathrm{DM}}$ is the Dzyaloshinskii-Moriya vector controlling the anti-symmetric part; the $\Theta$ is a traceless, symmetric $3 \times 3$ matrix. However, for further considerations, to describe manifolds of states, we shall use the explicit $\sigma$-matrix notation. The above interaction Hamiltonian is covered by the general form of a nonlocal Hamiltonian for a two-qubit system

$$
H=\sum_{i, j=1}^{3} h_{i j} \sigma_{i} \otimes \sigma_{j},
$$

$h_{i j}$ are real and $\sigma_{j}, j=1,2,3$ are Pauli matrices. As it is known [34], such a Hamiltonian can be transformed into the diagonal form

$$
H_{\mathrm{int}}=\sum_{j=1}^{3} c_{j} \sigma_{j} \otimes \sigma_{j} .
$$

The full Hamiltonian contains additionally a local term $H_{\text {loc }}=H_{1} \otimes \mathbb{1}+\mathbb{1} \otimes H_{2}$, with $H_{a}, a=1,2$ being one-qubit Hamiltonians. For simplicity, in the present work, we shall fix two-qubit local Hamiltonian in the form of the coupling of both systems to an external magnetic field along third axis, i.e.,

$$
H_{\mathrm{loc}}=H_{0} \equiv b\left(\sigma_{3} \otimes \mathbb{1}+\mathbb{1} \otimes \sigma_{3}\right)
$$

In the following, we shall consider unitary evolutions generated by four-parameter family of Hamiltonians 


$$
H\left(b, c_{1}, c_{2}, c_{3}\right)=H_{0}(b)+H_{\mathrm{int}}\left(c_{1}, c_{2}, c_{3}\right)
$$

The description of quantum state manifold and its metric geometry is crucial for answering many questions regarding the study of quantum evolution and in the information geometry approach, such as the determination of the volume of particular classes of states [18]. In [1], it was shown that the distance traveled by the system during the quantum evolution along a given curve in the projective Hilbert space is related to the speed of evolution. Using the symmetry properties of quantum state spaces, one can study the brachistochrone problem [22] and the time-optimal evolutions, e.g., using geometric properties of the quantum state manifolds of the spin- $s$ systems $[4,14,32]$, one can obtain conditions for the time-optimal quantum evolution of spin- $s$ in the magnetic field $[23,25]$. For example, for the systems of qubits and qutrits the optimal quantum circuit is equivalent to the shortest path between two points on the Riemannian manifold [5-8]. Moreover, the geometry of quantum state manifolds of the two-spin system interacting with the magnetic field, described by Heisenberg and Dzyaloshinskii-Moriya Hamiltonians with anisotropy along the one direction, is of great interest $[16,17]$. In this case, the related manifolds turn out to be flat and depend on the initial state and anisotropy parameter. In the present paper, we generalize this approach and consider the two-spin system with anisotropy in all directions of the magnetic field.

The organization of the paper is as follows. In the next section, we obtain the explicit parametrizations of sets of quantum states generated by evolution of selected initial states and obtain relevant manifolds of the dimension depending on the initial states. We describe the Riemannian geometry of the obtained manifolds by introducing the relevant Fubini-Study metrics for each case. What is important, we focus on the question of control of such systems. Therefore, assuming the final time of evolution as a fixed $t_{\text {fin }}$ (for convenience we put $t_{\text {fin }}=1$ ), we analyze the dependence of above manifolds and their geometry on the physical parameters entering the definition of the Hamiltonian. In Sect. 4, we study the changes in geometry resulting from a small perturbation in the original Hamiltonian by switching on an additional weak magnetic field along the first axis. Furthermore, in Sect. 5 we discuss the behavior of entanglement of states originating from the obtained manifolds using concurrence as the entanglement measure [35]. The concurrence changing from 0 to 1 in all cases indicates that it is not the intrinsic geometry of the manifold of quantum states and its dimension that determine the behavior of entanglement, but rather it is the specific location of such manifold relative to the torus $S^{2} \times S^{2}$ of the separable states inside the $S^{7}$.

\section{Unitary transformation of two-qubit state}

Let a unitary evolution be defined by the Hamiltonian $H$ given by Eq. (5) describing a two-qubit system with the anisotropic Heisenberg-type Hamiltonian in the magnetic field directed along the $z$-axis, i.e.,

$$
H=b\left(\sigma_{3} \otimes \mathbb{1}+\mathbb{1} \otimes \sigma_{3}\right)+\sum_{j=1}^{3} c_{j} \sigma_{j} \otimes \sigma_{j},
$$


where parameters $c_{j}, j=1,2,3$ are dimensionless interaction couplings between qubits and $b$ is a dimensionless parameter describing an external magnetic field [36].

This Hamiltonian has four eigenvalues: $E_{1}^{(0)}=c_{3}+\omega, E_{2}^{(0)}=c_{3}-\omega, E_{3}^{(0)}=$ $-c_{3}+c_{+}$, and $E_{4}^{(0)}=-c_{3}-c_{+}$, where $\omega=\sqrt{(2 b)^{2}+c_{-}^{2}}, \tan \phi=2 b / c_{-}$and $c_{ \pm}=c_{1} \pm c_{2}$. The corresponding eigenvectors have the following form

$$
\begin{aligned}
\left|\psi_{1}^{(0)}\right\rangle & =\frac{1}{\sqrt{2}}\left[\frac{\cos \phi}{\sqrt{1-\sin \phi}}|\uparrow \uparrow\rangle+\sqrt{1-\sin \phi}|\downarrow \downarrow\rangle\right], \\
\left|\psi_{2}^{(0)}\right\rangle & =\frac{1}{\sqrt{2}}\left[\frac{\cos \phi}{\sqrt{1+\sin \phi}}|\uparrow \uparrow\rangle-\sqrt{1+\sin \phi}|\downarrow \downarrow\rangle\right], \\
\left|\psi_{3}^{(0)}\right\rangle & =\frac{1}{\sqrt{2}}(|\uparrow \downarrow\rangle+|\downarrow \uparrow\rangle), \\
\left|\psi_{4}^{(0)}\right\rangle & =\frac{1}{\sqrt{2}}(|\uparrow \downarrow\rangle-|\downarrow \uparrow\rangle) .
\end{aligned}
$$

The unitary transformation $U\left(b, c_{j}\right)=U\left(\omega, \phi, c_{ \pm}\right)$, generated by the Hamiltonian (6), acts on an arbitrary quantum state of two qubits

$$
\left|\psi_{I}^{(0)}\right\rangle=\eta_{1}\left|\psi_{1}^{(0)}\right\rangle+\eta_{2}\left|\psi_{2}^{(0)}\right\rangle+\eta_{3}\left|\psi_{3}^{(0)}\right\rangle+\eta_{4}\left|\psi_{4}^{(0)}\right\rangle
$$

as follows

$$
\begin{gathered}
\left|\psi^{(0)}\left(\omega, \phi, c_{ \pm}\right)\right\rangle=U\left(\omega, \phi, c_{ \pm}\right)\left|\psi_{I}^{(0)}\right\rangle \\
\left|\psi^{(0)}\left(\omega, \phi, c_{ \pm}\right)\right\rangle=\mathrm{e}^{-i c_{3}}\left(\eta_{1} \mathrm{e}^{-i \omega}\left|\psi_{1}^{(0)}\right\rangle+\eta_{2} \mathrm{e}^{i \omega}\left|\psi_{2}^{(0)}\right\rangle\right. \\
\left.+\eta_{3} \mathrm{e}^{i\left(2 c_{3}-c_{+}\right)}\left|\psi_{3}^{(0)}\right\rangle+\eta_{4} \mathrm{e}^{i\left(2 c_{3}+c_{+}\right)}\left|\psi_{4}^{(0)}\right\rangle\right),
\end{gathered}
$$

where we 'freeze' evolution at $t=1$ to study the shape of manifolds determined by changes in parameters defining the Hamiltonian (6). It is worth noting that these parameters can define at most a four-dimensional manifold. Changing them in allowed ranges, determined by the periodic conditions of the state (12), we can cover this manifold. The time-evolution of the system governed by the Hamiltonian (6) with fixed values of the parameters $\left(\omega, \phi, c_{3}, c_{+}\right)$proceeds along a compact orbit passing through this manifold. The particular location of this orbit depends on the system parameters.

The normalization of an initial state means that $\left|\eta_{1}\right|^{2}+\left|\eta_{2}\right|^{2}+\left|\eta_{3}\right|^{2}+\left|\eta_{4}\right|^{2}=1$. Moreover, the state (12) depends on four parameters $\left(\omega, \phi, c_{3}, c_{+}\right)$satisfying some periodic conditions. These conditions, in turn, depend on the initial state coordinates $\eta_{j}$.

Let us classify the possible parametrizations as follows:

C1. For $\eta_{1}=\eta_{2}=0$ and $\eta_{3} \neq 0, \eta_{4} \neq 0$, the state (12) takes the form

$$
\left|\psi^{(0)}\right\rangle=\mathrm{e}^{i c_{3}}\left(\eta_{3} \mathrm{e}^{-i c_{+}}\left|\psi_{3}^{(0)}\right\rangle+\eta_{4} \mathrm{e}^{i c_{+}}\left|\psi_{4}^{(0)}\right\rangle\right) .
$$


It is easy to see that this state depends only on $c_{+}$parameter and satisfies the following periodic condition

$$
\left|\psi^{(0)}\left(c_{+}+\pi\right)\right\rangle=-\left|\psi^{(0)}\left(c_{+}\right)\right\rangle
$$

C2. For $\eta_{3}=\eta_{4}=0$ and $\eta_{1}=0$ or $\eta_{2}=0$, we obtain the states (7) or (8), which depend only on $\phi$ parameter with periodic condition

$$
\left|\psi^{(0)}(\phi+2 \pi)\right\rangle=\left|\psi^{(0)}(\phi)\right\rangle
$$

C3. For $\eta_{3}=\eta_{4}=0$ and nonzero $\eta_{1}, \eta_{2}$, the family of states is defined by the parameters $\omega$ and $\phi$ as follows

$$
\left|\psi^{(0)}\right\rangle=\mathrm{e}^{-i c_{3}}\left(\eta_{1} \mathrm{e}^{-i \omega}\left|\psi_{1}^{(0)}\right\rangle+\eta_{2} \mathrm{e}^{i \omega}\left|\psi_{2}^{(0)}\right\rangle\right)
$$

with the following periodic conditions

$$
\begin{aligned}
\left|\psi^{(0)}(\omega+\pi, \phi)\right\rangle & =-\left|\psi^{(0)}(\omega, \phi)\right\rangle, \\
\left|\psi^{(0)}(\omega, \phi+2 \pi)\right\rangle & =\left|\psi^{(0)}(\omega, \phi)\right\rangle .
\end{aligned}
$$

C4. For $\eta_{1}=0$ or $\eta_{2}=0$ and $\eta_{3}=0$ or $\eta_{4}=0$, the family of states is defined by two parameters $\phi$ and $c$, i.e.,

$$
\left|\psi^{(0)}\right\rangle=\mathrm{e}^{-i\left(c_{3}+(-1)^{l+1} \omega\right)}\left(\eta_{l}\left|\psi_{l}^{(0)}\right\rangle+\eta_{j} \mathrm{e}^{i c}\left|\psi_{j}^{(0)}\right\rangle\right)
$$

where $c=2 c_{3}+(-1)^{j} c_{+}+(-1)^{l+1} \omega$. Here, $l=1,2, j=3,4$. The states satisfy the following periodic conditions

$$
\begin{aligned}
& \left|\psi^{(0)}(\phi+2 \pi, c)\right\rangle=\left|\psi^{(0)}(\phi, c)\right\rangle, \\
& \left|\psi^{(0)}(\phi, c+2 \pi)\right\rangle=\left|\psi^{(0)}(\phi, c)\right\rangle .
\end{aligned}
$$

C5. If $\eta_{1}, \eta_{2}$ are nonzero, and $\eta_{3}=0$ or $\eta_{4}=0$, then the family of states is defined by three parameters: $\omega, \phi$ and $c$. Namely,

$$
\left|\psi^{(0)}\right\rangle=\mathrm{e}^{-i c_{3}}\left(\eta_{1} \mathrm{e}^{-i \omega}\left|\psi_{1}^{(0)}\right\rangle+\eta_{2} \mathrm{e}^{i \omega}\left|\psi_{2}^{(0)}\right\rangle+\eta_{j} \mathrm{e}^{i c}\left|\psi_{j}^{(0)}\right\rangle\right)
$$

Here, $c=2 c_{3}+(-1)^{j} c_{+}$. In this case, the states satisfy the following periodic conditions

$$
\begin{aligned}
& \left|\psi^{(0)}(\omega+\pi, \phi, c+\pi)\right\rangle=-\left|\psi^{(0)}(\omega, \phi, c)\right\rangle, \\
& \left|\psi^{(0)}(\omega, \phi+2 \pi, c)\right\rangle=\left|\psi^{(0)}(\omega, \phi, c)\right\rangle \\
& \left|\psi^{(0)}(\omega, \phi, c+2 \pi)\right\rangle=\left|\psi^{(0)}(\omega, \phi, c)\right\rangle .
\end{aligned}
$$


C6. For $\eta_{1}=0$ or $\eta_{2}=0$, and nonvanishing $\eta_{3}, \eta_{4}$, the family of states is defined by three parameters

$$
\left|\psi^{(0)}\right\rangle=\mathrm{e}^{-i\left(c_{3}+(-1)^{l+1} \omega\right)}\left(\eta_{l}\left|\psi_{l}^{(0)}\right\rangle+\eta_{3} \mathrm{e}^{i\left(c-c_{+}\right)}\left|\psi_{3}^{(0)}\right\rangle+\eta_{4} \mathrm{e}^{i\left(c+c_{+}\right)}\left|\psi_{4}^{(0)}\right\rangle\right) .
$$

Here, $c=2 c_{3}+(-1)^{l+1} \omega$. In this case, we have the following periodic conditions

$$
\begin{aligned}
& \left|\psi^{(0)}\left(\phi+2 \pi, c, c_{+}\right)\right\rangle=\left|\psi^{(0)}\left(\phi, c, c_{+}\right)\right\rangle, \\
& \left|\psi^{(0)}\left(\phi, c+2 \pi, c_{+}\right)\right\rangle=\left|\psi^{(0)}\left(\phi, c, c_{+}\right)\right\rangle, \\
& \left|\psi^{(0)}\left(\phi, c+\pi, c_{+}+\pi\right)\right\rangle=\left|\psi^{(0)}\left(\phi, c, c_{+}\right)\right\rangle .
\end{aligned}
$$

C7. In the general case, when all parameters $\eta_{1}, \eta_{2}, \eta_{3}$ and $\eta_{4}$ are nonzero, we have the state defined by expression (12) with the following periodic conditions

$$
\begin{aligned}
& \left|\psi^{(0)}\left(\omega+\pi, \phi, c_{3}+\pi / 2, c_{+}\right)\right\rangle=i\left|\psi^{(0)}\left(\omega, \phi, c_{3}, c_{+}\right)\right\rangle, \\
& \left|\psi^{(0)}\left(\omega+\pi, \phi, c_{3}, c_{+}+\pi\right)\right\rangle=-\left|\psi^{(0)}\left(\omega, \phi, c_{3}, c_{+}\right)\right\rangle, \\
& \left|\psi^{(0)}\left(\omega, \phi+2 \pi, c_{3}, c_{+}\right)\right\rangle=\left|\psi^{(0)}\left(\omega, \phi, c_{3}, c_{+}\right)\right\rangle, \\
& \left|\psi^{(0)}\left(\omega, \phi, c_{3}+\pi, c_{+}\right)\right\rangle=-\left|\psi^{(0)}\left(\omega, \phi, c_{3}, c_{+}\right)\right\rangle, \\
& \left|\psi^{(0)}\left(\omega, \phi, c_{3}+\pi / 2, c_{+}+\pi\right)\right\rangle=-i\left|\psi^{(0)}\left(\omega, \phi, c_{3}, c_{+}\right)\right\rangle .
\end{aligned}
$$

The above quantum state manifolds related to the unitary evolutions generated by the time-independent family of Hamiltonians are obviously closed. In the first two cases, quantum state manifold is one parameter, in cases three and four it is two-parameter manifold, in the fifth and sixth cases the manifold is defined by three parameters, and in the last case we have the four-parameter manifold.

Let us study the Fubini-Study metric of these manifolds, $\mathcal{M}_{\psi^{(0)}}$

\section{The Fubini-Study metric of quantum state manifolds}

The Fubini-Study metric is defined by the infinitesimal distance $d s$ between two neighboring pure quantum states $\left|\psi\left(\xi^{\mu}\right)\right\rangle$ and $\left|\psi\left(\xi^{\mu}+d \xi^{\mu}\right)\right\rangle$ [4]

$$
d s^{2}=g_{\mu \nu} d \xi^{\mu} d \xi^{\nu}
$$

where $\xi^{\mu}$ is a set of real parameters which define the state $\left|\psi\left(\xi^{\mu}\right)\right\rangle$. The components of the metric tensor $g_{\mu \nu}$ have the form

$$
g_{\mu \nu}=\gamma^{2} \Re\left(\left\langle\psi_{\mu} \mid \psi_{\nu}\right\rangle-\left\langle\psi_{\mu} \mid \psi\right\rangle\left\langle\psi \mid \psi_{\nu}\right\rangle\right)
$$

where $\gamma$ is an arbitrary factor which is often chosen to have value of $1, \sqrt{2}$ or 2 and

$$
\left|\psi_{\mu}\right\rangle=\frac{\partial}{\partial \xi^{\mu}}|\psi\rangle .
$$


As we have previously noted, the states (12) are defined by four real parameters. Using definition (26), we obtain the components of the metric tensor with respect to parameters $\left(\omega, \phi, c_{3}, c_{+}\right)$

$$
\begin{aligned}
& g_{\omega \omega}^{(0)}=\gamma^{2}\left(\eta_{12}^{+}-\left(\eta_{12}^{-}\right)^{2}\right), \quad g_{\omega \phi}^{(0)}=\gamma^{2} \eta_{12}^{-} J, \quad g_{\omega c_{3}}^{(0)}=2 \gamma^{2} \eta_{12}^{-} \eta_{34}^{+}, \\
& g_{\omega c_{+}}^{(0)}=-\gamma^{2} \eta_{12}^{-} \eta_{34}^{-}, \quad g_{\phi \phi}^{(0)}=\gamma^{2}\left(\frac{1}{4} \eta_{12}^{+}-J^{2}\right), \quad g_{\phi c_{3}}^{(0)}=-2 \gamma^{2} J \eta_{34}^{+} \\
& g_{\phi c_{+}}^{(0)}=\gamma^{2} J \eta_{34}^{-}, \quad g_{c_{3} c_{3}}^{(0)}=4 \gamma^{2} \eta_{12}^{+} \eta_{34}^{+}, \\
& g_{c_{3} c_{+}}^{(0)}=-2 \gamma^{2} \eta_{12}^{+} \eta_{34}^{-}, \quad g_{c_{+} c_{+}}^{(0)}=\gamma^{2}\left(\eta_{34}^{+}-\left(\eta_{34}^{-}\right)^{2}\right),
\end{aligned}
$$

where $\eta_{i j}^{ \pm}=\left|\eta_{i}\right|^{2} \pm\left|\eta_{j}\right|^{2}, J=\Im\left(\eta_{1} \eta_{2}^{*} \mathrm{e}^{-2 i \omega}\right)$. From the explicit form of the metric tensor, we see that in the case of the magnetic field switched off, one of the parameters disappears $(\phi=0)$ and the manifold becomes flat. It is the result of the reciprocal commutativity of the interaction terms in the Hamiltonian (6). It is worth noting that if $c_{1}=c_{2}$ and $c_{3}=\alpha c_{+} / 2$ then $\phi=\pi / 2$ and we obtain the metric of the two-parameter manifold as in [17]

$$
\begin{aligned}
g_{\omega \omega}^{(0)} & =\gamma^{2}\left(\eta_{12}^{+}-\left(\eta_{12}^{-}\right)^{2}\right), \quad g_{\omega c_{+}}^{(0)}=\gamma^{2} \eta_{12}^{-}\left(\alpha \eta_{34}^{+}-\eta_{34}^{-}\right), \\
g_{c_{+} c_{+}}^{(0)} & =\gamma^{2} \alpha \eta_{12}^{+}\left(\alpha \eta_{34}^{+}-2 \eta_{34}^{-}\right)+\gamma^{2}\left(\eta_{34}^{+}-\left(\eta_{34}^{-}\right)^{2}\right),
\end{aligned}
$$

where $\alpha$ is some real number that determines the anisotropy of the system. If $\alpha=1$, then we obtain the Fubini-Study metric of the quantum state manifold of isotropic Heisenberg model [16]. The metric (28) can be reduced to the diagonal form with the use of the new parameters after the following transformation

$$
\omega=\omega^{\prime}, \quad \phi=k_{1} \omega^{\prime}+\phi^{\prime}, \quad c_{3}=k_{2} \omega^{\prime}+k_{3} \phi^{\prime}+c_{3}^{\prime}, \quad c_{+}=k_{4} c_{3}^{\prime}+c_{+}^{\prime},
$$

where $\left.k_{1}=4 \eta_{12}^{-} J /\left(4 J^{2}-\left(\eta_{12}^{+}\right)^{2}\right), k_{2}=\eta_{12}^{+} \eta_{12}^{-} /\left(8 J^{2}-2\left(\eta_{12}^{+}\right)^{2}\right), k_{3}=J /\left(2 \eta_{12}^{+}\right)\right)$, $k_{4}=2 \eta_{12}^{+} \eta_{34}^{-} /\left(\eta_{34}^{+}-\left(\eta_{34}^{-}\right)^{2}\right)$. Let us additionally assume that

$$
J=\frac{\eta_{12}^{+}}{2} \cos \theta
$$

Then, in these new parameters, the metric (28) takes the following form

$$
\begin{aligned}
g_{\theta \theta}^{(0)} & =\frac{\gamma^{2}}{4} \eta_{12}^{+}, \quad g_{\phi^{\prime} \phi^{\prime}}^{(0)}=\frac{\gamma^{2}}{4} \eta_{12}^{+} \sin ^{2} \theta, \\
g_{c_{3}^{\prime} c_{3}^{\prime}}^{(0)} & =4 \gamma^{2} \eta_{12}^{+} \frac{\left(\eta_{34}^{+}\right)^{2}-\left(\eta_{34}^{-}\right)^{2}}{\eta_{34}^{+}-\left(\eta_{34}^{-}\right)^{2}}, \quad g_{c_{+}^{\prime} c_{+}^{\prime}}^{(0)}=\gamma^{2}\left(\eta_{34}^{+}-\left(\eta_{34}^{-}\right)^{2}\right) .
\end{aligned}
$$

It is evident that the ratio between the parameters of the initial state influences the components of the metric tensor. For instance, if $\eta_{3}=\eta_{4}$, then $\eta_{34}^{-}=0$ and $g_{c_{+}^{\prime} c_{+}^{\prime}}$ 
takes the maximal value for the specific initial state. Let us analyze in detail the geometry of the manifold defined by the above metric for the cases considered in the previous section:

1. In the first case, the manifold is defined by the parameter $c_{+} \in[0, \pi]$ and metric tensor is reduced to $g_{c_{+} c_{+}}$component with $\eta_{34}^{+}=1$. This is the metric of the circle of the radius $\gamma \sqrt{1-\left(\eta_{34}^{-}\right)^{2}} / 2$.

2. In the second case, the manifold is defined by parameter $\phi \in[0,2 \pi]$ and metric tensor is reduced to $g_{\phi \phi}^{(0)}$ with $\eta_{12}^{+}=1$. This metric also describes the circle of the radius $\gamma / 2$.

3. In this case, the manifold is two-parametric $\theta \in[0, \pi], \phi^{\prime} \in[0,2 \pi]$ and is described by the metric tensor with components $g_{\theta \theta}^{(0)}, g_{\phi^{\prime} \phi^{\prime}}^{(0)}$, where $\eta_{12}^{+}=1$. This means that it is the sphere of radius $\gamma / 2$.

4. Here, we have also two-parametric manifold defined by parameters $\phi \in[0,2 \pi]$, $c \in[0,2 \pi]$ and described by the following metric tensor

$$
g_{\phi \phi}^{(0)}=\frac{\gamma^{2}}{4}\left|\eta_{l}\right|^{2}, \quad g_{\phi c}^{(0)}=0, \quad g_{c c}^{(0)}=\gamma^{2}\left|\eta_{l}\right|^{2}\left|\eta_{j}\right|^{2} .
$$

As we can see, the components of the metric tensor do not depend on the parameters $\phi$ and $c$. This means that the manifold is flat. Taking into account periodic conditions (17), we conclude that it is a torus.

5. In the fifth case, the manifold is three-parametric and defined by the parameters $\theta \in[0, \pi], \phi^{\prime} \in[0,2 \pi], c^{\prime} \in[0,2 \pi]$. In the diagonal form, the metric tensor components $g_{\theta \theta}^{(0)}$, and $g_{\phi^{\prime} \phi^{\prime}}^{(0)}$ are defined by expression (32) and other component takes the form

$$
g_{c^{\prime} c^{\prime}}^{(0)}=\gamma^{2} \eta_{12}^{+}\left|\eta_{j}\right|^{2}
$$

where $c^{\prime}$ is related to the parameter $c$ from (20) by the following formula

$$
c=-\frac{\eta_{12}^{-}}{\eta_{12}^{+} \sin ^{2} \theta} \omega^{\prime}+\frac{1}{2} \cos \theta \phi^{\prime}+c^{\prime} .
$$

The manifold, which we obtain here, is a product of the sphere of radius $\gamma \sqrt{\eta_{12}^{+}} / 2$ in parameters $\theta, \phi^{\prime}$ and of the circle of radius $\gamma \sqrt{\eta_{12}^{+}}\left|\eta_{j}\right|$ in parameter $c^{\prime}$.

6. In the case C6, we obtain a manifold with the metric tensor in the diagonal form

$$
g_{\phi \phi}^{(0)}=\frac{\gamma^{2}}{4}\left|\eta_{l}\right|^{2}, \quad g_{c^{\prime} c^{\prime}}^{(0)}=\gamma^{2}\left|\eta_{l}\right|^{2} \frac{\left(\eta_{34}^{+}\right)^{2}-\left(\eta_{34}^{-}\right)^{2}}{\eta_{34}^{+}-\left(\eta_{34}^{-}\right)^{2}},
$$

and the component $g_{c_{+}^{\prime} c_{+}^{\prime}}^{(0)}$ defined by the expression (32). Therefore, we obtain a three-parameter manifold defined by $\phi \in[0,2 \pi], c^{\prime} \in[0,2 \pi], c_{+}^{\prime} \in[0, \pi]$. To diagonalize this metric, we use the following transformation 


$$
c=c^{\prime}, \quad c_{+}=\frac{\left|\eta_{l}\right|^{2} \eta_{34}^{-}}{\eta_{34}^{+}-\left(\eta_{34}^{-}\right)^{2}} c^{\prime}+c_{+}^{\prime},
$$

where $c$ is defined as for the state (22). So, this manifold can be expressed by a circle of radius $\gamma\left|\eta_{l}\right| / 2$ in parameter $\phi$ and torus in parameter $c^{\prime}, c_{+}^{\prime}$.

7. In the general case, the metric is defined by expression (28) or (32). This manifold consists of two submanifolds, namely a sphere of radius $\gamma \sqrt{\eta_{12}^{+}} / 2$ in parameters $\theta \in[0, \pi], \phi^{\prime} \in[0,2 \pi]$ and a torus in parameters $c_{3}^{\prime} \in[0, \pi], c_{+}^{\prime} \in[0, \pi]$.

\section{The Fubini-Study metric of quantum state manifold with perturbation}

In this section, we study the Fubini-Study metric of quantum state manifold obtained as a result of actions of unitary transformations generated by the Hamiltonian (6) modified by an additional perturbation term which do not commute with the original Hamiltonian. This perturbation switches on a weak magnetic field directed along the $x$-axis with value $\beta$, where $\beta$ is assumed to be small. Explicitly, the Hamiltonian of this system takes the form

$$
H^{\prime}=H+\beta\left(\sigma_{x} \otimes I+I \otimes \sigma_{x}\right)
$$

We find the eigenvalues and eigenstates of this Hamiltonian using the perturbation theory with respect to the first order of $\beta$. So, the eigenvalues of Hamiltonian (38) are the same as for Hamiltonian (6), which correspond to the following eigenstates

$$
\begin{aligned}
\left|\psi_{1}\right\rangle= & \left|\psi_{1}^{(0)}\right\rangle+\frac{1}{\sqrt{2}} \frac{\beta}{2 c_{3}+\omega-c_{+}} \frac{\cos \phi-\sin \phi+1}{\sqrt{1-\sin \phi}}[|\uparrow \downarrow\rangle+|\downarrow \uparrow\rangle], \\
\left|\psi_{2}\right\rangle= & \left|\psi_{2}^{(0)}\right\rangle+\frac{1}{\sqrt{2}} \frac{\beta}{2 c_{3}-\omega-c_{+}} \frac{\cos \phi-\sin \phi-1}{\sqrt{1+\sin \phi}}[|\uparrow \downarrow\rangle+|\downarrow \uparrow\rangle], \\
\left|\psi_{3}\right\rangle= & \left|\psi_{3}^{(0)}\right\rangle+\frac{\beta}{-2 c_{3}-\omega+c_{+}} \frac{\cos \phi-\sin \phi+1}{\sqrt{2}}\left[\frac{\cos \phi}{1-\sin \phi}|\uparrow \uparrow\rangle+|\downarrow \downarrow\rangle\right] \\
& +\frac{\cos \phi-\sin \phi-1}{\sqrt{2}} \frac{\beta}{-2 c_{3}+\omega+c_{+}}\left[\frac{\cos \phi}{1+\sin \phi}|\uparrow \uparrow\rangle-|\downarrow \downarrow\rangle\right], \\
\left|\psi_{4}\right\rangle= & \left|\psi_{4}^{(0)}\right\rangle .
\end{aligned}
$$

Similar to the previous case, we decompose the initial state using the eigenstates (39). Then, the evolution is described by equation (12) with the above eigenstates. The Fubini-Study metric for evolution generated by the linearly perturbed Hamiltonian takes the following form

$$
\begin{aligned}
g_{\omega \omega}= & g_{\omega \omega}^{(0)}+2 \beta \gamma^{2}\left\{\left(1-2 \eta_{12}^{-}\right) \Im\left(\eta_{1} \eta_{3}^{*} \mathrm{e}^{-i\left(2 c_{3}+\omega-c_{+}\right)}\right) Y_{+}\right. \\
& \left.+\left(1+2 \eta_{12}^{-}\right) \Im\left(\eta_{2} \eta_{3}^{*} \mathrm{e}^{-i\left(2 c_{3}-\omega-c_{+}\right)}\right) Y_{-}\right\},
\end{aligned}
$$




$$
\begin{aligned}
& g_{c_{3} c_{3}}=g_{c_{3} c_{3}}^{(0)}-8 \beta \gamma^{2}\left(\eta_{12}^{+}-\eta_{34}^{+}\right)\left\{\mathfrak{s}\left(\eta_{1} \eta_{3}^{*} \mathrm{e}^{-i\left(2 c_{3}+\omega-c_{+}\right)}\right) Y_{+}\right. \\
& \left.+\mathfrak{I}\left(\eta_{2} \eta_{3}^{*} \mathrm{e}^{-i\left(2 c_{3}-\omega-c_{+}\right)}\right) Y_{-}\right\} \text {, } \\
& g_{c_{+} c_{+}}=g_{c_{+} c_{+}}^{(0)}-2 \beta \gamma^{2}\left(1-2 \eta_{34}^{-}\right)\left\{\mathfrak{s}\left(\eta_{1} \eta_{3}^{*} \mathrm{e}^{-i\left(2 c_{3}+\omega-c_{+}\right)}\right) Y_{+}\right. \\
& \left.+\mathfrak{I}\left(\eta_{2} \eta_{3}^{*} \mathrm{e}^{-i\left(2 c_{3}-\omega-c_{+}\right)}\right) Y_{-}\right\} \text {, } \\
& g_{\phi \phi}=g_{\phi \phi}^{(0)}+\beta \gamma^{2} \omega\left\{\left(4 J \Im\left(\eta_{1} \eta_{3}^{*} \mathrm{e}^{-i\left(2 c_{3}+\omega-c_{+}\right)}\right)-\Re\left(\eta_{2} \eta_{3}^{*} \mathrm{e}^{-i\left(2 c_{3}-\omega-c_{+}\right)}\right)\right) X_{-}\right. \\
& \left.+\left(4 J \Im\left(\eta_{2} \eta_{3}^{*} \mathrm{e}^{-i\left(2 c_{3}-\omega-c_{+}\right)}\right)+\Re\left(\eta_{1} \eta_{3}^{*} \mathrm{e}^{-i\left(2 c_{3}+\omega-c_{+}\right)}\right)\right) X_{+}\right\}, \\
& g_{\phi \omega}=g_{\phi \omega}^{(0)}+\beta \gamma^{2}\left\{\omega\left(1-\eta_{12}^{-}\right) \mathfrak{s}\left(\eta_{1} \eta_{3}^{*} \mathrm{e}^{-i\left(2 c_{3}+\omega-c_{+}\right)}\right) X_{-}\right. \\
& -\omega\left(1+\eta_{12}^{-}\right) \Im\left(\eta_{2} \eta_{3}^{*} \mathrm{e}^{-i\left(2 c_{3}-\omega-c_{+}\right)}\right) X_{+} \\
& -\left(\frac{1}{2} \Re\left(\eta_{1} \eta_{3}^{*} \mathrm{e}^{-i\left(2 c_{3}+\omega-c_{+}\right)}\right)+2 J \Im\left(\eta_{2} \eta_{3}^{*} \mathrm{e}^{-i\left(2 c_{3}-\omega-c_{+}\right)}\right)\right) Y_{-} \\
& \left.-\left(\frac{1}{2} \Re\left(\eta_{2} \eta_{3}^{*} \mathrm{e}^{-i\left(2 c_{3}-\omega-c_{+}\right)}\right)-2 J \Im\left(\eta_{1} \eta_{3}^{*} \mathrm{e}^{-i\left(2 c_{3}+\omega-c_{+}\right)}\right)\right) Y_{+}\right\}, \\
& g_{c_{3} \omega}=g_{c_{3} \omega}^{(0)}+4 \beta \gamma^{2}\left\{\left(\eta_{34}^{+}-\eta_{12}^{-}\right) \Im\left(\eta_{1} \eta_{3}^{*} \mathrm{e}^{-i\left(2 c_{3}+\omega-c_{+}\right)}\right) Y_{+}\right. \\
& \left.-\left(\eta_{34}^{+}+\eta_{12}^{-}\right) \Im\left(\eta_{2} \eta_{3}^{*} \mathrm{e}^{-i\left(2 c_{3}-\omega-c_{+}\right)}\right) Y_{-}\right\} \text {, } \\
& g_{c_{+} \omega}=g_{c_{+} \omega}^{(0)}+2 \beta \gamma^{2}\left\{\left(\eta_{12}^{-}-\eta_{34}^{-}\right) \mathfrak{s}\left(\eta_{1} \eta_{3}^{*} \mathrm{e}^{-i\left(2 c_{3}+\omega-c_{+}\right)}\right) Y_{+}\right. \\
& \left.+\left(\eta_{12}^{-}+\eta_{34}^{-}\right) \Im\left(\eta_{2} \eta_{3}^{*} \mathrm{e}^{-i\left(2 c_{3}-\omega-c_{+}\right)}\right) Y_{-}\right\}, \\
& g_{c_{3} \phi}=g_{c_{3} \phi}^{(0)} \\
& +\beta \gamma^{2}\left\{-2 \omega\left(\eta_{12}^{+}-\eta_{34}^{+}\right)\left(\Im\left(\eta_{1} \eta_{3}^{*} \mathrm{e}^{-i\left(2 c_{3}+\omega-c_{+}\right)}\right) X_{-}\right.\right. \\
& \left.+\mathfrak{s}\left(\eta_{2} \eta_{3}^{*} \mathrm{e}^{-i\left(2 c_{3}-\omega-c_{+}\right)}\right) X_{+}\right) \\
& +\left(\Re\left(\eta_{1} \eta_{3}^{*} \mathrm{e}^{-i\left(2 c_{3}+\omega-c_{+}\right)}\right)+4 J \Im\left(\eta_{2} \eta_{3}^{*} \mathrm{e}^{-i\left(2 c_{3}-\omega-c_{+}\right)}\right)\right) Y_{-} \\
& \left.-\left(\Re\left(\eta_{2} \eta_{3}^{*} \mathrm{e}^{-i\left(2 c_{3}-\omega-c_{+}\right)}\right)-4 J \Im\left(\eta_{1} \eta_{3}^{*} \mathrm{e}^{-i\left(2 c_{3}+\omega-c_{+}\right)}\right)\right) Y_{+}\right\}, \\
& g_{c_{+} \phi}=g_{c_{+} \phi}^{(0)} \\
& +\beta \gamma^{2}\left\{\omega ( 1 - 2 \eta _ { 3 4 } ^ { - } ) \left(\Im\left(\eta_{1} \eta_{3}^{*} \mathrm{e}^{-i\left(2 c_{3}+\omega-c_{+}\right)}\right) X_{-}\right.\right. \\
& \left.+\Im\left(\eta_{2} \eta_{3}^{*} \mathrm{e}^{-i\left(2 c_{3}-\omega-c_{+}\right)}\right) X_{+}\right) \\
& -\left(\frac{1}{2} \Re\left(\eta_{1} \eta_{3}^{*} \mathrm{e}^{-i\left(2 c_{3}+\omega-c_{+}\right)}\right)+2 J \Im\left(\eta_{2} \eta_{3}^{*} \mathrm{e}^{-i\left(2 c_{3}-\omega-c_{+}\right)}\right)\right) Y_{-} \\
& \left.+\left(\frac{1}{2} \Re\left(\eta_{2} \eta_{3}^{*} \mathrm{e}^{-i\left(2 c_{3}-\omega-c_{+}\right)}\right)-2 J \Im\left(\eta_{1} \eta_{3}^{*} \mathrm{e}^{-i\left(2 c_{3}+\omega-c_{+}\right)}\right)\right) Y_{+}\right\} \text {, }
\end{aligned}
$$


Table 1 Dimensions and parametrization of the state manifolds

\begin{tabular}{lll}
\hline Case & $\operatorname{dim} \mathcal{M}_{\left|\psi^{(0)}\right\rangle}$ & Parameters \\
\hline $\mathrm{C} 1$ & 1 & $c_{+}$ \\
$\mathrm{C} 2$ & 1 & $\phi$ \\
$\mathrm{C} 3$ & 2 & $\omega, \phi$ \\
$\mathrm{C} 4$ & 2 & $\phi, c$ \\
$\mathrm{C} 5$ & 3 & $\omega, \phi, c$ \\
$\mathrm{C} 6$ & 3 & $\phi, c, c_{+}$ \\
$\mathrm{C} 7$ & 4 & $\omega, \phi, c_{3} c_{+}$ \\
\hline
\end{tabular}

$$
\begin{aligned}
g_{c_{+} c_{3}}= & g_{c_{+} c_{3}}^{(0)}+2 \beta \gamma^{2}\left(2 \eta_{12}^{+}-\eta_{34}^{-}\right)\left\{\Im\left(\eta_{1} \eta_{3}^{*} \mathrm{e}^{-i\left(2 c_{3}+\omega-c_{+}\right)}\right) Y_{+}\right. \\
& \left.+\Im\left(\eta_{2} \eta_{3}^{*} \mathrm{e}^{-i\left(2 c_{3}-\omega-c_{+}\right)}\right) Y_{-}\right\},
\end{aligned}
$$

where we use the following notation

$$
Y_{ \pm}=\frac{\sqrt{1-\sin (\phi)} \pm \sqrt{1+\sin (\phi)}}{\left(2 c_{3}-c_{+} \pm \omega\right)^{2}}, \quad X_{ \pm}=\frac{\sqrt{1-\sin (\phi)} \pm \sqrt{1+\sin (\phi)}}{\left(2 c_{3}-c_{+}\right)^{2}-\omega^{2}}
$$

and the above components give the new perturbed metric of the form

$$
g_{i j}=g_{i j}^{(0)}+\beta h_{i j}
$$

Such perturbation modifies geometry of some of the state manifolds enlisted in Table 1. For the cases $\mathrm{C} 1$ to $\mathrm{C} 3$, there is no modification at all. The cases $\mathrm{C} 4$ and $\mathrm{C} 5$ for choices $\eta_{4} \neq 0$ are also unperturbed. A nontrivial modification appears for C6 and C7. The explicit formulas for the scalar curvature of perturbed metric for these cases are hard to obtain. To illustrate the effect of modification on the manifold, let us consider special initial conditions for the case C7. Let us assume that $\left|\eta_{1}\right|=\left|\eta_{2}\right|=\left|\eta_{3}\right|=\left|\eta_{4}\right|=\frac{1}{2}$. Then, the metric (28) takes the following form

$$
g^{(0)}=\left(\begin{array}{cccc}
\frac{\gamma^{2}}{2} & 0 & 0 & 0 \\
0 & \frac{1}{32} \gamma^{2}\left(\cos \left(2\left(\alpha_{12}+2 \omega\right)\right)+3\right) & \frac{1}{4} \gamma^{2} \sin \left(\alpha_{12}+2 \omega\right) & 0 \\
0 & \frac{1}{4} \gamma^{2} \sin \left(\alpha_{12}+2 \omega\right) & \gamma^{2} & 0 \\
0 & 0 & 0 & \frac{\gamma^{2}}{2}
\end{array}\right)
$$

and yields the Ricci tensor

$$
\mathcal{R}=\left(\begin{array}{cccc}
3 & 0 & 0 & 0 \\
0 & \frac{1}{16}\left(5 \cos \left(2\left(\alpha_{12}+2 \omega\right)\right)+7\right) & \frac{1}{2} \sin \left(\alpha_{12}+2 \omega\right) & 0 \\
0 & \frac{1}{2} \sin \left(\alpha_{12}+2 \omega\right) & 2 & 0 \\
0 & 0 & 0 & 0
\end{array}\right)
$$


where $\eta_{j}=\left|\eta_{j}\right| \mathrm{e}^{i \alpha_{j}}, \alpha_{i j}=\alpha_{i}-\alpha_{j}$ and the scalar curvature $R=\frac{14}{\gamma^{2}}$. Here, parameters $\phi, c_{+}, c_{3}, \alpha_{13}$ and $\alpha_{23}$ do not influence the value of $R$, one can also put $\alpha_{12}=0$. Now, for the perturbed metric (50) depending solely on $\omega$, we get the following scalar curvature

$$
R=\frac{14}{\gamma^{2}} \frac{\cos (2 \omega)}{14(\cos (4 \omega)+1)^{2}}\left(\frac{A_{1}}{A_{2}}+\frac{B_{1}}{B_{2}}+\frac{C_{1}}{C_{2}}+\frac{D_{1}}{D_{2}}+\frac{E_{1}}{E_{2}}\right)
$$

where functions entering the above formula have the form

$$
\begin{aligned}
& A_{1}=8 \beta \cos ^{4}(2 \omega)\left(4 \omega^{2} \sin (\omega)+6\left(\omega^{2}-2\right) \sin \omega \cos (2 \omega)+8 \omega \cos (3 \omega)\right), \\
& A_{2}=6 \beta \omega^{2}(4 \omega \sin (\omega)+\sin (\omega)+\sin (3 \omega)-4 \omega \cos (\omega)+\cos (\omega) \\
& -\cos (5 \omega))+\omega^{4}+\omega^{4} \cos (4 \omega), \\
& B_{1}=(\cos (4 \omega)+1)\left(\beta \left(-16 \omega^{2} \sin (\omega)+16 \omega^{2} \sin (3 \omega)\right.\right. \\
& -2\left(8 \omega^{2}+3 \omega-12\right) \cos (\omega)-4\left(4 \omega^{2}+9 \omega-1\right) \cos (3 \omega) \\
& +5 \omega \sin (\omega)+5 \omega \sin (3 \omega)+\omega \sin (5 \omega)+\omega \sin (7 \omega)-4 \sin (\omega) \\
& +24 \sin (3 \omega)+4 \sin (7 \omega)-6 \omega \cos (7 \omega)+4 \cos (5 \omega)) \\
& \left.+7 \omega^{3} \cos (2 \omega)+\omega^{3} \cos (6 \omega)\right) \\
& B_{2}=4 \beta \omega(4 \omega \sin (\omega)+\sin (\omega)+\sin (3 \omega)-4 \omega \cos (\omega)+\cos (\omega) \\
& -\cos (5 \omega))+\omega^{3}+\omega^{3} \cos (4 \omega), \\
& C_{1}=2 \sin (4 \omega)\left(2 \beta\left(14 \omega^{3}+4 \omega^{2}-7 \omega+6\right) \cos (\omega)-2 \beta\left(14 \omega^{3} \sin (\omega)\right.\right. \\
& +10 \omega^{3} \sin (3 \omega)-12 \omega^{2} \sin (\omega)+7 \omega^{2} \sin (3 \omega)+7 \omega^{2} \sin (7 \omega) \\
& +\omega^{2} \cos (7 \omega)+\left(4 \omega^{2}-3\right) \cos (5 \omega)+\left(10 \omega^{3}+5 \omega^{2}+12 \omega-3\right) \cos (3 \omega) \\
& -\omega \sin (\omega)-\omega \sin (7 \omega)+3 \sin (\omega)-6 \sin (3 \omega)-3 \sin (7 \omega) \\
& \left.+5 \omega \cos (7 \omega))+\omega^{4} \sin (2 \omega)+\omega^{4} \sin (6 \omega)\right), \\
& C_{2}=6 \beta \omega^{2}(4 \omega \sin (\omega)+\sin (\omega)+\sin (3 \omega)-4 \omega \cos (\omega)+\cos (\omega) \\
& -\cos (5 \omega))+\omega^{4}+\omega^{4} \cos (4 \omega), \\
& D_{1}=\cos (2 \omega)\left(\beta \left(160 \omega^{2} \sin (\omega)-152 \omega^{2} \sin (3 \omega)+104 \omega^{2} \sin (5 \omega)-104 \omega^{2} \cos (5 \omega)\right.\right. \\
& -2\left(80 \omega^{2}+9 \omega-32\right) \cos (\omega)+\left(-152 \omega^{2}+62 \omega+40\right) \cos (3 \omega)+52 \omega \sin (\omega) \\
& -46 \omega \sin (3 \omega)+42 \omega \sin (5 \omega)+19 \omega \sin (7 \omega)+7 \omega \sin (9 \omega)+16 \sin (3 \omega) \\
& +16 \sin (5 \omega)-54 \omega \cos (5 \omega)-22 \omega \cos (9 \omega)+24 \cos (5 \omega)-4 \cos (7 \omega) \\
& \left.+4 \cos (9 \omega))+19 \omega^{3}+24 \omega^{3} \cos (4 \omega)+5 \omega^{3} \cos (8 \omega)\right) \\
& D_{2}=6 \beta \omega(4 \omega \sin (\omega)+\sin (\omega)+\sin (3 \omega)-4 \omega \cos (\omega)+\cos (\omega)-\cos (5 \omega)) \\
& +\omega^{3}+\omega^{3} \cos (4 \omega) \\
& E_{1}=\cos (2 \omega)\left(-2 \beta\left(40 \omega^{3}+21 \omega^{2}-44 \omega+6\right) \cos (\omega)+2 \beta\left(-100 \omega^{3}+55 \omega^{2}\right.\right. \\
& +36 \omega-6) \cos (3 \omega)+\beta\left(80 \omega^{3} \sin (\omega)-200 \omega^{3} \sin (3 \omega)+136 \omega^{3} \sin (5 \omega)\right. \\
& +24 \omega^{2} \sin (\omega)-82 \omega^{2} \sin (3 \omega)+54 \omega^{2} \sin (5 \omega)
\end{aligned}
$$




$$
\begin{aligned}
& +25 \omega^{2} \sin (7 \omega)+9 \omega^{2} \sin (9 \omega)+\left(-38 \omega^{2}+8 \omega+12\right) \cos (9 \omega) \\
& -2\left(68 \omega^{3}+31 \omega^{2}-12 \omega-6\right) \cos (5 \omega)-16 \omega \sin (\omega)+48 \omega \sin (3 \omega) \\
& +48 \omega \sin (5 \omega)+16 \omega \sin (9 \omega)-48 \sin (\omega)+12 \sin (3 \omega)-36 \sin (5 \omega) \\
& \left.-6 \sin (7 \omega)-6 \sin (9 \omega))+18 \omega^{4}+24 \omega^{4} \cos (4 \omega)+6 \omega^{4} \cos (8 \omega)\right) \\
E_{2}= & 6 \beta \omega^{2}(4 \omega \sin (\omega)+\sin (\omega)+\sin (3 \omega)-4 \omega \cos (\omega)+\cos (\omega)-\cos (5 \omega)) \\
& +\omega^{4}+\omega^{4} \cos (4 \omega)
\end{aligned}
$$

Let us note that switching the perturbation off (53) gives the correct unperturbed scalar curvature.

\section{Entanglement characterization of two-qubit quantum state manifolds $\mathcal{M}_{\left|\psi^{(0)}\right\rangle}$}

In the present section, using the squared concurrence as an entanglement measure, we shall study the entanglement of states belonging to the manifolds obtained in Sect. 2. For a pure state of bipartite two-level system, it is defined as follows $[35,37,38]$

$$
C(|\psi\rangle) \equiv 2|a d-b c|
$$

where $a, b, c$ and $d$ are defined by expression

$$
|\psi\rangle=a|\uparrow \uparrow\rangle+b|\uparrow \downarrow\rangle+c|\downarrow \uparrow\rangle+d|\downarrow \downarrow\rangle .
$$

The squared concurrence for state (12) takes the form

$$
\begin{aligned}
C\left(\left|\psi_{0}\right\rangle\right)=\mid & \left(\eta_{1}^{2} \mathrm{e}^{-2 i \omega}-\eta_{2}^{2} \mathrm{e}^{2 i \omega}\right) \cos \phi \\
& -2 \eta_{1} \eta_{2} \sin \phi-\mathrm{e}^{4 i c_{3}}\left(\eta_{3}^{2} \mathrm{e}^{-2 i c_{+}}-\eta_{4}^{2} \mathrm{e}^{2 i c_{+}}\right) \mid .
\end{aligned}
$$

Let us calculate the squared concurrence for the families of states discussed in the previous sections:

1. In the case $\mathrm{C} 1$, the concurrence takes the form

$$
C=\sqrt{\left|\eta_{3}\right|^{4}+\left|\eta_{4}\right|^{4}-2\left|\eta_{3}\right|^{2}\left|\eta_{4}\right|^{2} \cos \left(4 c_{+}+2 \chi\right)}
$$

For $\eta_{3}=\left|\eta_{3}\right|$ and $\eta_{4}=\left|\eta_{4}\right| \mathrm{e}^{i \chi}$, where $\chi \in[0,2 \pi]$. We obtain the maximally entangled state if $c_{+}=1 / 4[(2 n+1) \pi-2 \chi]$, where $n \in \mathbb{Z}$.

2. The squared concurrence in the case $\mathrm{C} 2$ takes simple form

$$
C=|\cos \phi|
$$


3. For the $\mathrm{C} 3$ family of states, the manifold is defined by two parameters. The entanglement of the states is described by the following expression

$$
\begin{gathered}
C=\left[\left(\left|\eta_{1}\right|^{4}+\left|\eta_{2}\right|^{4}-2\left|\eta_{1}\right|^{2}\left|\eta_{2}\right|^{2} \cos (4 \omega+2 \chi)\right) \cos ^{2} \phi+4\left|\eta_{1}\right|^{2}\left|\eta_{2}\right|^{2} \sin ^{2} \phi\right. \\
\left.-4\left|\eta_{1}\right|\left|\eta_{2}\right|\left(\left|\eta_{1}\right|^{2}-\left|\eta_{2}\right|^{2}\right) \cos (2 \omega+\chi) \sin \phi \cos \phi\right]^{1 / 2}
\end{gathered}
$$

Similarly, as in the previous case $\mathrm{C} 1$, we put $\eta_{1}=\left|\eta_{1}\right|$ and $\eta_{2}=\left|\eta_{2}\right| \mathrm{e}^{i \chi}$. As we can see, regardless of the initial state, the maximally entangled state is obtained when $\phi=0$ and $\omega=1 / 4[(2 n+1) \pi-2 \chi]$.

4. Here, we also put $\eta_{l}=\left|\eta_{l}\right|$ and $\eta_{j}=\left|\eta_{j}\right| \mathrm{e}^{i \chi}$ and obtain the expression for concurrence

$$
C=\sqrt{\left|\eta_{l}\right|^{4} \cos ^{2} \phi+\left|\eta_{j}\right|^{4}-2(-1)^{l+j}\left|\eta_{l}\right|^{2}\left|\eta_{j}\right|^{2} \cos (2 c+2 \chi) \cos \phi} .
$$

So, the conditions for maximally entangled are the following: $\phi=0$ and $c=$ $1 / 2[(2 n+1) \pi-2 \chi]$ for even $l+j, c=1 / 2[2 \pi n-2 \chi]$ for odd $l+j$, and, respectively, for $\phi=\pi$.

5. For the C5 family of states, to simplify calculations, we analyze the case when $\eta_{1}=\eta_{2}$ and we put $\eta_{1}=\left|\eta_{1}\right|, \eta_{j}=\left|\eta_{j}\right| \mathrm{e}^{i \chi}$. The squared concurrence takes finally the form

$$
\begin{aligned}
C= & {\left[\left(-2\left|\eta_{1}\right|^{2} \sin \phi+(-1)^{j}\left|\eta_{j}\right|^{2} \cos (2 c+2 \chi)\right)^{2}\right.} \\
& \left.+\left(-2\left|\eta_{1}\right|^{2} \sin 2 \omega \cos \phi+(-1)^{j}\left|\eta_{j}\right|^{2} \sin (2 c+2 \chi)\right)^{2}\right]^{1 / 2} .
\end{aligned}
$$

We collect the conditions for preparation of maximally entangled states in Table 2.

6. We shall use similar simplifications in the case C6. Here, we also put $\eta_{3}=\eta_{4}$ and $\eta_{l}=\left|\eta_{l}\right|, \eta_{3}=\left|\eta_{3}\right| \mathrm{e}^{i \chi}$. Then, the squared concurrence takes the form

$$
\begin{aligned}
C= & {\left[\left((-1)^{l+1}\left|\eta_{l}\right|^{2} \cos \phi-2\left|\eta_{3}\right|^{2} \sin (2 c+2 \chi) \sin 2 c_{+}\right)^{2}\right.} \\
& \left.+4\left|\eta_{3}\right|^{4} \cos ^{2}(2 c+2 \chi) \sin ^{2} 2 c_{+}\right]^{1 / 2}
\end{aligned}
$$

The conditions for preparation of maximally entangled states for the C6-family are presented in Table 3.

7. In the C7 case, we assume that $\eta_{1}=\eta_{2}=\left|\eta_{1}\right|, \eta_{3}=\eta_{4}=\left|\eta_{3}\right| \mathrm{e}^{i \chi}$ what yields the squared concurrence in the form

$$
\begin{aligned}
C= & {\left[\left(2\left|\eta_{1}\right|^{2} \sin \phi+2\left|\eta_{3}\right|^{2} \sin 2 c_{+} \sin \left(4 c_{3}+2 \chi\right)\right)^{2}\right.} \\
& \left.+\left(-2\left|\eta_{1}\right|^{2} \sin 2 \omega \cos \phi+2\left|\eta_{3}\right|^{2} \sin 2 c_{+} \cos \left(4 c_{3}+2 \chi\right)\right)^{2}\right]^{1 / 2}
\end{aligned}
$$


Table 2 Conditions for maximally entangled states in case C5

Table 3 Conditions for maximally entangled states in the case $\mathrm{C} 6$

\begin{tabular}{llll}
\hline$\phi$ & $\omega$ & $j$ & $c$ \\
\hline 0 & $\pi / 4$ & Even & $3 \pi / 4+\pi n-\chi$ \\
& $3 \pi / 4$ & Odd & $\pi / 4+\pi n-\chi$ \\
& & Even & $\pi / 4+\pi n-\chi$ \\
$\pi / 2$ & Odd & $3 \pi / 4+\pi n-\chi$ \\
& - & Even & $1 / 2[(2 n+1) \pi-2 \chi]$ \\
$\pi$ & & Odd & $\pi n-\chi$ \\
& $\pi / 4$ & Even & $\pi / 4+\pi n-\chi$ \\
& & Odd & $3 \pi / 4+\pi n-\chi$ \\
& $3 \pi / 4$ & Even & $3 \pi / 4+\pi n-\chi$ \\
& & Odd & $\pi / 4+\pi n-\chi$ \\
& - & Even & $\pi n-\chi$ \\
& & Odd & $1 / 2[(2 n+1) \pi-2 \chi]$ \\
\hline
\end{tabular}

\begin{tabular}{llll}
\hline$\phi$ & $l$ & $c$ & $c_{+}$ \\
\hline 0 & Even & $\pi / 4+\pi n-\chi$ & $\pi / 4+\pi n$ \\
& & $3 \pi / 4+\pi n-\chi$ & $3 \pi / 4+\pi n$ \\
& Odd & $\pi / 4+\pi n-\chi$ & $3 \pi / 4+\pi n$ \\
& & $3 \pi / 4+\pi n-\chi$ & $\pi / 4+\pi n$ \\
$\pi$ & Even & $\pi / 4+\pi n-\chi$ & $3 \pi / 4+\pi n$ \\
& & $3 \pi / 4+\pi n-\chi$ & $\pi / 4+\pi n$ \\
& & $\pi / 4+\pi n-\chi$ & $\pi / 4+\pi n$ \\
& & $3 \pi / 4+\pi n-\chi$ & $3 \pi / 4+\pi n$ \\
\hline
\end{tabular}

The conditions defining maximally entangled states are collected in Table 4.

It is worth noting that in all above cases, one can achieve full range of values of the concurrence. This effect indicates that it is not the geometry and dimensionality of the particular state manifold that is decisive, rather it is the location of such manifold within the $S^{7}$ and its common points with the torus $S^{2} \times S^{2}$ of separable pure state manifold. For states with vanishing concurrence for special choices of the relevant parameters, we can talk about a 'parametric death' of entanglement-paraphrasing the 'sudden death' of entanglement discussed in the literature for specific evolutions. However, having in mind the entangling power of the considered families of Hamiltonians we have collected conditions for preparation of maximally entangled states for all cases under consideration.

\section{Conclusions}

The geometric characterization of the state manifold of quantum system is of great value, but for compound systems such task becomes very complex when addressed in the general setting. 
Table 4 Conditions for maximally entangled states in case C7

\begin{tabular}{llll}
\hline$\phi$ & $\omega$ & $c+$ & $c_{3}$ \\
\hline 0 & $\pi / 4$ & $\pi / 4+\pi n$ & $1 / 4[(2 n+1) \pi-2 \chi]$ \\
& $3 \pi / 4+\pi n$ & $1 / 2[\pi n-\chi]$ \\
& $3 \pi / 4$ & $\pi / 4+\pi n$ & $1 / 2[\pi n-\chi]$ \\
& & $3 \pi / 4+\pi n$ & $1 / 4[(2 n+1) \pi-2 \chi]$ \\
$\pi / 2$ & - & $\pi / 4+\pi n$ & $1 / 4[\pi / 2+2 \pi n-2 \chi]$ \\
& & $3 \pi / 4+\pi n$ & $1 / 4[3 \pi / 2+2 \pi n-2 \chi]$ \\
$\pi$ & $\pi / 4$ & $\pi / 4+\pi n$ & $1 / 2[\pi n-\chi]$ \\
& & $3 \pi / 4+\pi n$ & $1 / 4[(2 n+1) \pi-2 \chi]$ \\
& $3 \pi / 4$ & $\pi / 4+\pi n$ & $1 / 4[(2 n+1) \pi-2 \chi]$ \\
& & $3 \pi / 4+\pi n$ & $1 / 2[\pi n-\chi]$ \\
$3 \pi / 2$ & - & $\pi / 4+\pi n$ & $1 / 4[3 \pi / 2+2 \pi n-2 \chi]$ \\
& & $3 \pi / 4+\pi n$ & $1 / 4[\pi / 2+2 \pi n-2 \chi]$ \\
\hline
\end{tabular}

In the present work, we have studied quantum state manifolds for two-qubit system obtained by means of the unitary evolution defined by large family of physically interesting Hamiltonians. Despite the knowledge of the whole set of the two-qubit quantum states, it is important to know what manifolds lying inside this set can be reached using the evolution governed by the realistic Hamiltonians. The geometry of such obtained compact quantum state spaces is of Riemannian type defined by the Fubini-Study metrics depending on initial conditions and parameters entering the definition of the families of Hamiltonians. We have given the classification of possible state manifolds and thoroughly discussed the explicit description of two-qubit unitary orbits generated by physically relevant Hamiltonians. The relevant FubiniStudy metrics were obtained with the use of the explicit parametrizations.

It is worth noting that we have also studied the question of how obtained geometries are modified by the noncommutative linear perturbation term included into the original Hamiltonian. We describe its influence on the scalar curvature of the relevant state spaces. In some cases, the answer turns out to be nontrivial.

As an important physical characterization of the considered systems, we have studied the degree of entanglement of states for all obtained quantum state spaces and we have provided conditions for producing maximally entangled states in each case, where the concurrence was used as an entanglement monotone. Such knowledge seems to be of great importance for the quantum information and quantum computation applications.

Acknowledgements One of the authors (A.K.) wishes to thank the Institute of Theoretical Physics at the University of Wroclaw for hospitality and financial support as well as he acknowledges that the work was supported by Project FF-30F (No. 0116U001539) from the Ministry of Education and Science of Ukraine.

Open Access This article is distributed under the terms of the Creative Commons Attribution 4.0 International License (http://creativecommons.org/licenses/by/4.0/), which permits unrestricted use, distribution, and reproduction in any medium, provided you give appropriate credit to the original author(s) and the source, provide a link to the Creative Commons license, and indicate if changes were made. 


\section{References}

1. Anandan, J., Aharonov, Y.: Geometry of quantum evolution. Phys. Rev. Lett. 65, 1697 (1990)

2. Abe, S.: Quantized geometry associated with uncertainty and correlation. Phys. Rev. A 48, 4102 (1993)

3. Laba, H.P., Tkachuk, V.M.: Geometric characteristics of quantum evolution: curvature and torsion. Condens. Matter Phys. 20, 13003 (2017)

4. Bengtsson, I., Życzkowski, K.: Geometry of Quantum States. Cambridge University Press, New York (2006)

5. Nielsen, M.A., Dowling, M.R., Gu, M., Doherty, A.C.: Optimal control, geometry, and quantum computing. Phys. Rev. A 73, 062323 (2006)

6. Nielsen, M.A.: A geometric approach to quantum circuit lower bounds. Quantum Inf. Comput. 6, 213 (2006)

7. Nielsen, M.A., Dowling, M.R., Gu, M., Doherty, A.C.: Quantum computation as geometry. Science 311, 1133 (2006)

8. Khaneja, N., Heitmann, B., Spörl, A., Yuan, H., Schulte-Herbrüggen, T., Glaser, S.J.: Quantum gate design metric. arXiv:quant-ph/0605071 (2006)

9. Barenco, A., Bennett, C.H., Cleve, R., DiVincenzo, D.P., Margolus, N., Shor, P., Sleator, T., Smolin, J.A., Weinfurter, H.: Elementary gates for quantum computation. Phys. Rev. A. 52, 3457 (1995)

10. Kane, B.E.: A silicon-based nuclear spin quantum computer. Nature (London) 393, 133 (1998)

11. DiVincenzo, D.P., Bacon, D., Kempe, J., Burkard, G., Whaley, K.B.: Universal quantum computation with the exchange interaction. Nature 408, 339 (2000)

12. Hanson, A.J., Ortiz, G., Sabry, A., Tai, Yu-Tsung: Geometry of discrete quantum computing. J. Phys. A 46, 185301 (2013)

13. Kolodrubetz, M., Gritsev, V., Polkovnikov, A.: Classifying and measuring geometry of a quantum ground state manifold. Phys. Rev. B 88, 064304 (2013)

14. Brody, D.C., Graefe, E.-M.: Coherent states and rational surfaces. J. Phys. A 43, 255205 (2010)

15. Kuzmak, A.R.: Geometry of quantum state manifolds generated by the Lie algebra operators. J. Geom. Phys. 126, 1 (2018)

16. Kuzmak, A.R., Tkachuk, V.M.: Geometry of a two-spin quantum state in evolution. J. Phys. A 49, 045301 (2016)

17. Kuzmak, A.R.: Quantum state geometry and entanglement of two spins with anisotropic interaction in evolution. J. Geom. Phys. 116, 81 (2017)

18. Rexiti, M., Felice, D., Mancini, S.: The volume of two-qubit states by information geometry. Entropy 20, 146 (2018)

19. Avron, J.E., Kenneth, O.: Entanglement and the geometry of two qubits. Ann. Phys. 324, 470 (2009)

20. Palao, J.P., Kosloff, R.: Optimal control theory for unitary transformations. Phys. Rev. A 68, 062308 (2003)

21. Khaneja, N., Glaser, S.J., Brockett, R.: Sub-Riemannian geometry and time optimal control of three spin systems: quantum gates and coherence transfer. Phys. Rev. A 65, 032301 (2002)

22. Brody, D.C., Hook, D.W.: On optimum Hamiltonians for state transformations. J. Phys. A 39, L167 (2006)

23. Frydryszak, A.M., Tkachuk, V.M.: Quantum brachistochrone problem for a spin-1 system in a magnetic field. Phys. Rev. A 77, 014103 (2008)

24. Kuzmak, A.R., Tkachuk, V.M.: The quantum brachistochrone problem for two spins- $\frac{1}{2}$ with anisotropic Heisenberg interaction. J. Phys. A 46, 155305 (2013)

25. Kuzmak, A.R., Tkachuk, V.M.: The quantum brachistochrone problem for an arbitrary spin in a magnetic field. Phys. Lett. A 379, 1233 (2015)

26. Russell, B., Stepney, S.: Zermelo navigation in the quantum brachistochrone. J. Phys. A 48, 115303 (2015)

27. Brody, D.C., Gibbons, G.W., Meier, D.M.: Time-optimal navigation through quantum wind. New J. Phys. 17, 033048 (2015)

28. Brody, D.C., Meier, D.M.: Solution to the quantum Zermelo navigation problem. Phys. Rev. Lett. 114, $100502(2015)$

29. Abe, S.: Quantum-state space metric and correlations. Phys. Rev. A 46, 1667 (1992)

30. Page, D.N.: Geometrical description of Berry's phase. Phys. Rev. A 36, 3479(R) (1987)

31. Kobayashi, S., Nomizu, K.: Foundations of Differential Geometry, vol. 2. Wiley, New York (1969) 
32. Provost, J.P., Valle, G.: Riemannian structure on manifolds of quantum states. Commun. Math. Phys. 76, 289 (1980)

33. Revicule, M., Cassas, M., Plastino, A.: Information and metrics in Hilbert space. Phys. Rev. 55, 1695 (1997)

34. Zhang, J., Vala, J., Sastry, S., Whaley, K.B.: Geometric theory of nonlocal two-qubit operations. Phys. Rev. A 67, 042313 (2003)

35. Wootters, W.K.: Entanglemet of formation and concurrence. Quantum Inf. Comput. 1, 27 (2001)

36. Frydryszak, A.M.: Nilpotent quantum mechanics. Int. J. Mod. Phys. A 25, 951 (2010)

37. Wootters, W.K.: Entanglement of formation of an arbitrary state of two qubits. Phys. Rev. Lett. 80, 2245 (1998)

38. Hill, S., Wootters, W.K.: Entanglement of a pair of quantum bits. Phys. Rev. Lett. 78, 5022 (1997)

Publisher's Note Springer Nature remains neutral with regard to jurisdictional claims in published maps and institutional affiliations. 